\title{
Extending Genetic Linkage Analysis to Diffusion Tensor Images to Map Single Gene Effects on Brain Fiber Architecture*
}

\author{
Ming-Chang Chiang ${ }^{1}$, Christina Avedissian ${ }^{1}$, Marina Barysheva $^{1}$, Arthur W. Toga ${ }^{1}$, \\ Katie L. McMahon ${ }^{2}$, Greig I. de Zubicaray ${ }^{2}$, Margaret J. Wright ${ }^{3}$, \\ and Paul M. Thompson ${ }^{1}$ \\ ${ }^{1}$ Laboratory of Neuro Imaging, Dept. of Neurology, \\ UCLA School of Medicine, Los Angeles, CA \\ ${ }^{2}$ University of Queensland, Functional Magnetic Resonance Imaging Laboratory, \\ Centre for Magnetic Resonance, Brisbane, Australia \\ ${ }^{3}$ Queensland Institute of Medical Research, Brisbane, Australia
}

\begin{abstract}
We extended genetic linkage analysis - an analysis widely used in quantitati ve genetics - to $3 \mathrm{D}$ images to analyze single gene effects on brain fiber architecture. We collected 4 Tesla diffusion tensor images (DTI) and genotype data from 258 healthy adult twins and their non-twin siblings. After highdimensional fluid registration, at each voxel we estimated the genetic linkage between the single nucleotide polymorphism (SNP), Val66Met (dbSNP number rs6265), of the BDNF gene (brain-derived neurotrophic factor) with fractional anisotropy (FA) derived from each subject's DTI scan, by fitting structural equation models (SEM) from quantitative genetics. We also examined how image filtering affects the effect sizes for genetic linkage by examining how the overall significance of voxelwise effects varied with respect to full width at half maximum (FWHM) of the Gaussian smoothing applied to the FA images. Raw FA maps with no smoothing yielded the greatest sensitivity to detect gene effects, when corrected for multiple comparisons using the false discovery rate (FDR) procedure. The BDNF polymorphism significantly contributed to the variation in FA in the posterior cingulate gyrus, where it accounted for around $90-95 \%$ of the total variance in FA. Our study generated the first maps to visualize the effect of the BDNF gene on brain fiber integrity, suggesting that common genetic variants may strongly determine white matter integrity.
\end{abstract}

\section{Introduction}

Imaging genetics is a rapidly growing field that combines mathematical methods from image analysis and quantitative genetics to discover how specific genes influence the brain, cognition, and risk for disease. Although epidemiologists can relate genetic data to behavior in thousands of subjects, a more mechanistic understanding of these effects

* This work was funded in part by NIH grant R01 HD050735. 
is likely to be gained by linking genetic variants to specific features in images (such as measures of fiber integrity) that are highly correlated with intellectual performance and risk for disease, and may explain the basis of the genetic effect. Mapping these effects in $3 \mathrm{D}$ may also focus on specific regions where the genetic influences are stronger than elsewhere. This may also increase sensitivity for detecting genetic linkage because millions of hypotheses (voxelwise statistics) are tested simultaneously throughout the brain, while false discovery rate methods can still control the false positive rate. Structural MRI studies in twins have shown that many aspects of brain structure are highly heritable, including cortical gray matter density and thickness [1]. More recently, the first genetic studies of 3D diffusion tensor images have revealed that fiber integrity is under strong genetic control, is correlated with intelligence quotient (IQ) and the same genes underlie both fiber integrity and IQ [2]. These initial studies fitted quantitative genetic models to separate additive genetic from common and unique environmental components of inter-subject variance in images, for measures of fiber integrity such as the fractional anisotropy (FA) and generalized FA in high-angular resolution diffusion images (HARDI; [3]). McIntosh et al. [4] first studied the association between FA and individual genes, identifying the T-allele in a single nucleotide locus (dbSNP number rs6994992) of the neuregulin 1 gene as being associated with reduced FA in the anterior limb of the internal capsule.

In this paper we extended the variance-component genetic linkage analysis method [5] to create voxel-level maps in fluidly registered DTI data from a population, to estimate the influences of a specific gene on fiber integrity in the brain. A common variant in the brain-derived neurotrophic factor (BDNF) gene was linked with the variation in FA, an accepted measure of fiber integrity. BDNF modulates synaptic plasticity in the hippocampus, and is crucially involved in memory acquisition and retention. We selected the most studied single nucleotide polymorphism (SNP) of the BDNF gene, Val66Met (dbSNP number rs6265), where valine (Val) is substituted by methionine (Met) at codon 66 in the $5^{\prime}$-proregion of the BDNF protein. This Val66Met SNP has been found to be associated with subjects' memory performance and memory-related hippocampal activation using functional MRI [6]. We acquired 4 Tesla diffusion tensor images from 258 twins and their non-twin siblings, and fitted structural equation models (SEM; [5]) at each voxel to estimate the contribution of the BDNF Val66Met SNP to the variance in FA. After multiple comparisons correction using FDR, we still found significant linkage between this common BDNF polymorphism and FA in the posterior cingulate gyrus. To inform future genetic linkage studies in image databases, we performed a post hoc (exploratory) test to see how the overall significance of voxelwise gene effects varied with respect to the size of the Gaussian filter kernel applied to the FA images.

\section{Methods}

\subsection{Subject Description and Genotyping}

258 subjects (110 males/148 females; age: $23.8 \pm 1.9$ years, mean \pm SD), consisting of 39 monozygotic (MZ) twin pairs, 65 dizygotic (DZ) twin pairs, 1 set of DZ triplets, and 47 of their non-twin siblings, were recruited from 133 different nuclear families, as part of a 5-year research project evaluating healthy Australian twins using structural 
and functional MRI and DTI. The genotype of the BDNF Val66Met polymorphism was determined using primer extension on the Sequenom Mass-Array system [7], with $\mathrm{Val} / \mathrm{Val}$ identified in 174 subjects, Val/Met in 68 subjects, and Met/Met in 16 subjects. Information from a larger sample of subjects [7], which include all subjects in this study, has shown that the frequency of BDNF Val allele is 0.81, and that the genotype distribution follows Hardy-Weinberg equilibrium. This candidate gene was chosen based on prior reports that it explained variation in memory performance, task-related activation on fMRI, and its known molecular role as a key growth factor affecting brain development and plasticity [6].

\subsection{Image Acquisition and Registration}

All MR images were collected using a 4 Tesla Bruker Medspec MRI scanner. Diffusion-weighted scans were acquired using single-shot echo planar imaging with a twice-refocused spin echo sequence to reduce eddy-current induced distortions. Acquisition parameters were optimized to provide the best signal-to-noise ratio for estimation of diffusion tensors. Imaging parameters were: 21 axial slices ( $5 \mathrm{~mm}$ thick), FOV $=23 \mathrm{~cm}$, TR/TE $6090 / 91.7 \mathrm{~ms}, 0.5 \mathrm{~mm}$ gap, with a $128 \times 100$ acquisition matrix. 30 images were acquired: 3 with no diffusion sensitization (i.e., T2-weighted images) and 27 diffusion-weighted (DW) images $\left(b=1132 \mathrm{~s} / \mathrm{mm}^{2}\right)$ in which the gradient directions were evenly distributed on an imaginary hemisphere. The reconstruction matrix was $128 \times 128$, yielding a $1.8 \times 1.8 \mathrm{~mm}^{2}$ in-plane resolution. Total scan time was 3.05 minutes. We used the FMRIB software library (FSL, http://www.fmrib.ox. ac.uk/fsl/) for initial pre-processing of the diffusion images. For each subject, motion artifacts were corrected by linearly registering all the T2-weighted and DW images to one of the T2-weighted image (the "eddy_correct" command). Then the three T2weighted images were averaged and stripped of non-brain tissues to yield a binary brain extraction mask (cerebellum included), using the Brain Extraction Tool (BET) [8], followed by expert manual editing if necessary. The masked T2-weighted image was then registered to the ICBM standardized brain template with a 9-parameter linear transformation using the software FLIRT [9]. The resulting transformation parameters were used to rotationally reorient the diffusion tensors (computed from DW images using the "DTIFIT" command) at each voxel. The tensor-valued images were linearly realigned based on trilinear interpolation of the log-transformed tensors, and resampled to isotropic voxel resolution (with dimensions: $128 \times 128 \times 93$ voxels, resolution: $1.7 \times 1.7 \times 1.7 \mathrm{~mm}^{3}$ ). The fractional anisotropy (FA) image derived from the affine-registered DT image was then fluidly registered to a randomly selected subject's FA image, based on maximizing the Jensen-Rényi divergence (JRD) of the joint intensity histogram [10]. Here we preferred direct fluid alignment of FA, due to the need for computational efficiency in 258 subjects, although we are exploring alternative more CPU-intensive methods we developed to fluidly align diffusion tensors using information theory [11].

\subsection{Linkage between the BDNF Polymorphism and FA}

We used structural equation modeling [5] to analyze the linkage between BDNF polymorphism and brain diffusion anisotropy, in which the total variance of FA at 
each voxel was attributed to the additive genetic variance of the BDNF polymorphism ( $\sigma_{a}^{2}$; the variance due to the additive effect of the BDNF polymorphism), the residual genetic variance $\left(\sigma_{g}^{2}\right.$; the variance due to all other genes on the genome), the variance of the shared family rearing environment $\left(\sigma_{c}{ }^{2}\right)$, and the individual unique $\left(\sigma_{e}{ }^{2}\right)$ environmental factors. We note that linkage does not necessarily mean that the genetic marker (e.g. the BDNF Val66Met polymorphism here) directly causes a difference in an image, but rather indicates that the genetic marker is close to the genes that influence the phenotype. Genes close to each other on the genome tend to be inherited together, so one can genotype a set of specific genes to identify which genetic marker an influential polymorphism is close to, and in that case the genotyped gene is said to be in genetic linkage with the trait - here the FA of the image.

Because of the known genetic similarity between relatives of different kinds, it is possible to write the covariance matrix of FA for the $i$ th family, denoted by $\Phi_{i}$, in terms of the different sources of variance being modeled (namely, $\sigma_{a}{ }^{2}, \sigma_{g}{ }^{2}, \sigma_{c}{ }^{2}, \sigma_{e}{ }^{2}$ ), as follows:

$$
\Phi_{i j k}=\left\{\begin{array}{l}
\sigma_{a}^{2}+\sigma_{g}^{2}+\sigma_{c}^{2}+\sigma_{e}^{2}, \text { if } j=k, \text { or } j \text { and } k \text { are MZ twins } \\
\sigma_{a}^{2}+\sigma_{g}^{2}+\sigma_{c}^{2}, \text { if } j \neq k, \text { and } j \text { and } k \text { are MZ twins } \\
\pi_{i j k} \sigma_{a}^{2}+1 / 2 \sigma_{g}^{2}+\sigma_{c}^{2}, \text { if } j \neq k \text {, and } j \text { and } k \text { are not MZ twins, }
\end{array}\right.
$$

where $\pi_{i j k}$ is the expected proportion of alleles that arose from the same ancestor allele, i.e., identical by descent (IBD), for subjects $j$ and $k$. We estimated $\pi_{i j k}$ using the Haseman-Elston method detailed in [12]. The structural equation model in Eq (1) was fitted using the maximum-likelihood method [5], given by

$$
\log L=\sum_{i=1}^{N}-\frac{1}{2} \log \left|\Phi_{i}\right|-\frac{1}{2} y_{i}^{T} \Phi_{i}^{-1} y_{i},
$$

where the $n_{i}$-sample vector $y_{i}$ is the FA value of all $n_{i}$ subjects in family $i$, adjusted for the age and sex of each subject. $N$ denotes the number of families. $\sigma_{a}{ }^{2}, \sigma_{g}{ }^{2}, \sigma_{c}{ }^{2}$, and $\sigma_{e}^{2}$ were estimated using the Broyden-Fletcher-Goldfarb-Shanno (BFGS) method [13] to maximize $\log L$ in Eq (2). The significance of the influences of the BDNF genetic variation (polymorphism) was determined by the difference between the loglikelihood of the full model that included $\sigma_{a}{ }^{2}, \sigma_{g}{ }^{2}, \sigma_{c}{ }^{2}$, and $\sigma_{e}{ }^{2}$ and the restricted model including $\sigma_{g}{ }^{2}, \sigma_{c}^{2}$, and $\sigma_{e}^{2}$ only, denoted by $\log L_{f}$ for the full and $\log L_{r}$ for the restricted model. Minus two times this difference, or $-2\left(\log L_{r}-\log L_{f}\right)$, is asymptotically distributed approximately as a chi-squared distribution with one degree of freedom. The resulting statistics were plotted at each point in the image to show the voxelwise significance of the genetic linkage between the BDNF gene and FA in all 258 subjects. The overall significance of the resulting statistical maps was assessed using the false discovery rate (FDR) method [14] to correct for multiple comparisons. By convention, a FDR value $\leq 0.05$ was considered to be significant.

\section{Results}

Fig. 1 shows that 90-95\% variance of FA (with no smoothing; see below) in the posterior cingulate gyrus and right frontal area was attributable to the genetic influences of 

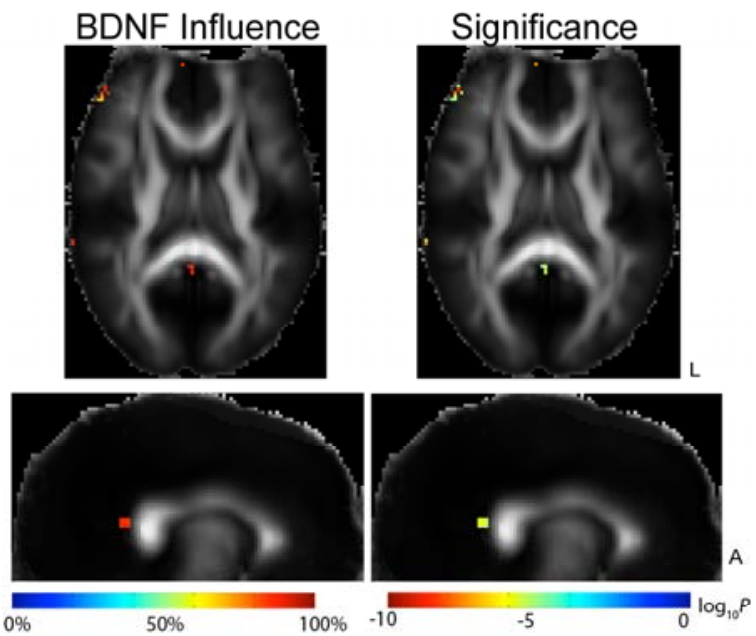

Fig. 1. Proportional contribution (left upper and lower panels) and its statistical significance (right upper and lower panels; the color scale is proportional to the decadic logarithm of the voxel $P$-value) of the BDNF polymorphism to the local variation in brain fiber integrity, measured by FA. The colored regions are the clusters composed of 26-connected voxels with $P$-values not greater than the threshold, computed by the FDR procedure that controls the expected false discovery rate to be no greater than $5 \%$. For better visualization, only clusters with 10 or more voxels are displayed. The BDNF polymorphism significantly contributes to $90-95 \%$ variance of FA mainly in the posterior cingulate gyrus and in the right frontal area. L: left hemisphere. A: anterior.

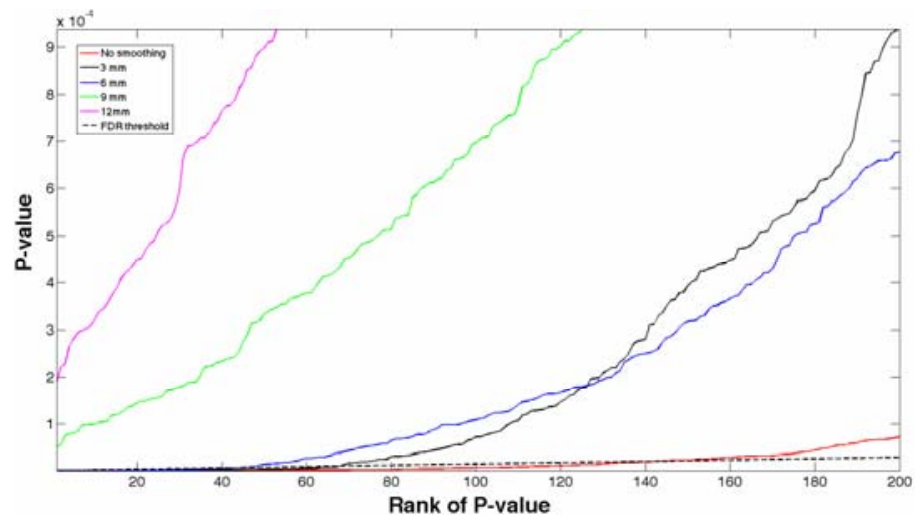

Fig. 2. Plot of the ordered voxel-level $P$-values showing the significance of the linkage between the BDNF polymorphism and FA smoothed using a 3D Gaussian kernel with different FWHM (in $\mathrm{mm}$ ). The $P$-value was computed by comparing the log-likelihood of the full versus the restricted structural equation models. $P$-values are considered significant overall when they are not greater than the threshold where the individual curve intersects the dashed line $y=0.05 \mathrm{x} / \mathrm{n}$, where $\mathrm{n}$ is the number of voxels within the brain-only mask; then the false discovery rate is controlled at no more than $5 \%$. 
the BDNF polymorphism. BDNF is known to be an important gene for long-term memory and learning, which may be enhanced by greater myelination and therefore axonal conduction speed in the cingulate gyrus, which is known to receive fibers from the subiculum of the hippocampus, which is involved in working memory.

To assess how different levels of smoothing influence the effect size of the linkage between the BDNF polymorphism and diffusion anisotropy, we also fitted the structural equation model [Eq (1)] with subjects' FA images (stripped of non-brain tissues) smoothed using a Gaussian filter with a full width at half maximum $(\mathrm{FWHM})=3,6$, 9, or $12 \mathrm{~mm}$. Fig. 2 displays the voxel-level $P$-values of the significance for the FABDNF linkage, plotted against their rank. FA with no smoothing yielded the greatest number of FDR-significant voxels - defined as voxels where the $P$-value was not greater than the threshold that controlled the FDR at the level of 0.05 , as also shown in Table 1. Even so, if we compared the smoothing effect based on the size of FDRsignificant clusters, which were sets of connected (26-neighborhood) FDR-significant voxels, FA that was moderately smoothed $(\mathrm{FWHM}=3$ and $6 \mathrm{~mm}$ ) yielded a single large cluster in the posterior cingulate gyrus, while FA with no smoothing yielded many tiny clusters that were one or two voxels in size (Table 1). As it is not legitimate to search over multiple filtered images to find effects, we report the unsmoothed data for purposes of statistical inference but we report the smoothed data as a post hoc assessment of the parameters that are likely to give best sensitivity in future analyses of independent (non-overlapping) data. Scale-space searches (i.e., over multiple filtered images) have been proposed in fMRI studies [15] although they are computationally intensive and can inflate type I error unless subjected to a global FDR procedure.

Table 1. The effect of FA smoothing on the detection of FA-BDNF linkage

$\left.\begin{array}{lccccc}\hline & 0 & \text { FWHM (mm) } & & \\ & 142 & 3 & 6 & 9 & 12 \\ \hline \begin{array}{l}\text { Number of FDR- } \\ \text { significant voxels }\end{array} & 1(18), 2(9), 3(5), 4(4), 5(1), & 1(1), 2(1), & 44(1) & & 0 \\ \begin{array}{l}\text { FDR-significant } \\ \text { clusters* }\end{array} & 10(1), 11(1), 13(1), 16(1), 20(1) & 7(1), 58(1)\end{array}\right)$

*Listed based on their size in voxels (the number of clusters of each size is in parentheses).

\section{Conclusion}

In this paper we first visualized the linkage between the BDNF Val66Met polymorphism and brain fiber architecture by analyzing the FA images of a large sample of twins and non-twin siblings. We also compared the effects of different magnitudes of image smoothing, and found that using raw FA images with no smoothing was most sensitive for detecting the FA-BDNF linkage when the FDR method was used to correct for multiple comparisons across the whole brain. The practical importance of this paper is that by finding a common gene variant that affects fiber integrity, measured by FA, one could (1) study whether it is over-represented in the many common diseases where FA is reduced, to provide a more mechanistic understanding of the disease, (2) co-vary for (adjust) its effects in studies of FA to improve statistical power, (3) look at the trajectory of FA over time in relation to the BDNF variant subgroups, 
and discover how the gene acts to affect fiber integrity, and (4) see if the BNDF variant confers increased risk for other deficits than FA (e.g. structural or functional deficits in the same regions).

Brain fiber integrity measured by FA is highly heritable, and higher FA is associated with better intellectual performance [2]. Our results show that BDNF is one of the genes that influence the fiber architecture in the posterior cingulate region. Both BDNF and the cingulate gyrus have key roles in mediating cognitive function, and future studies will aim to evaluate the cross-trait linkage of BDNF to FA in this region and to the subjects' cognitive function and intellectual performance.

\section{References}

1. Thompson, P.M., Cannon, T.D., Narr, K.L., van Erp, T., Poutanen, V.P., Huttunen, M., Lonnqvist, J., Standertskjold-Nordenstam, C.G., Kaprio, J., Khaledy, M., Dail, R., Zoumalan, C.I., Toga, A.W.: Genetic influences on brain structure. Nat. Neurosci. 4, 1253-1258 (2001)

2. Chiang, M.C., Barysheva, M., Shattuck, D.W., Lee, A.D., Madsen, S.K., Avedissian, C., Klunder, A.D., Toga, A.W., McMahon, K.L., de Zubicaray, G.I., Wright, M.J., Srivastava, A., Balov, N., Thompson, P.M.: Genetics of brain fiber architecture and intellectual performance. J. Neurosci. 29, 2212-2224 (2009)

3. Chiang, M.C., Barysheva, M., Lee, A.D., Madsen, S., Klunder, A.D., Toga, A.W., McMahon, K.L., de Zubicaray, G.I., Meredith, M., Wright, M.J., Srivastava, A., Balov, N., Thompson, P.M.: Brain fiber architecture, genetics, and intelligence: a high angular resolution diffusion imaging (HARDI) study. In: Metaxas, D., Axel, L., Fichtinger, G., Székely, G. (eds.) MICCAI 2008, Part I. LNCS, vol. 5241, pp. 1060-1067. Springer, Heidelberg (2008)

4. McIntosh, A.M., Moorhead, T.W., Job, D., Lymer, G.K., Munoz Maniega, S., McKirdy, J., Sussmann, J.E., Baig, B.J., Bastin, M.E., Porteous, D., Evans, K.L., Johnstone, E.C., Lawrie, S.M., Hall, J.: The effects of a neuregulin 1 variant on white matter density and integrity. Mol. Psychiatry 13, 1054-1059 (2008)

5. Almasy, L., Blangero, J.: Multipoint quantitative-trait linkage analysis in general pedigrees. Am. J. Hum. Genet. 62, 1198-1211 (1998)

6. Hariri, A.R., Goldberg, T.E., Mattay, V.S., Kolachana, B.S., Callicott, J.H., Egan, M.F., Weinberger, D.R.: Brain-derived neurotrophic factor val66met polymorphism affects human memory-related hippocampal activity and predicts memory performance. J. Neurosci. 23, 6690-6694 (2003)

7. Hansell, N.K., James, M.R., Duffy, D.L., Birley, A.J., Luciano, M., Geffen, G.M., Wright, M.J., Montgomery, G.W., Martin, N.G.: Effect of the BDNF V166M polymorphism on working memory in healthy adolescents. Genes. Brain Behav. 6, 260-268 (2007)

8. Smith, S.M.: Fast robust automated brain extraction. Hum. Brain Mapp. 17, 143-155 (2002)

9. Jenkinson, M., Smith, S.: A global optimisation method for robust affine registration of brain images. Med. Image Anal. 5, 143-156 (2001)

10. Chiang, M.-C., Dutton, R.A., Hayashi, K.M., Lopez, O.L., Aizenstein, H.J., Toga, A.W., Becker, J.T., Thompson, P.M.: 3D pattern of brain atrophy in HIV/AIDS visualized using tensor-based morphometry. Neuroimage 34, 44-60 (2007)

11. Chiang, M.C., Leow, A.D., Klunder, A.D., Dutton, R.A., Barysheva, M., Rose, S.E., McMahon, K.L., de Zubicaray, G.I., Toga, A.W., Thompson, P.M.: Fluid registration of diffusion tensor images using information theory. IEEE Trans. Med. Imaging 27, 442-456 (2008) 
12. Haseman, J.K., Elston, R.C.: The investigation of linkage between a quantitative trait and a marker locus. Behav. Genet. 2, 3-19 (1972)

13. Press, W.H., Teukolsky, S.A., Vetterling, W.T., Flannery, B.P.: Numerical recipes in C++. Cambridge Univ. Press, Cambridge (2002)

14. Benjamini, Y., Hochberg, Y.: Controlling the false discovery rate: a practical and powerful approach to multiple testing. Journal of the Royal Statistical Society. Series B (Methodological) 57, 289-300 (1995)

15. Siegmund, D.O., Worsley, K.J.: Testing for a signal with unknown location and scale in a stationary Gaussian random field. The Annals of Statistics 23, 608-639 (1995) 